\title{
UTILISATION DE LA COLCHICINE DANS L'ÉTUDE DES DIVISIONS GONIALES DE L'OVAIRE D'EMBRYONS DE BREBIS ET ANALYSE DE QUELQUES RÉSULTATS
}

\author{
P. MAULÉON \\ Avec la collaboration technique d'OLGa VolkorF \\ Station de Recherches de Physiologie animale, \\ Centre national de Recherches zootechniques. Jouy-en-Josas.
}

\section{SOMMAIRE}

La colchicine a été utilisée pour bloquer les divisions goniales de l'ovaire de Brebis entre le $35^{\mathrm{e}}$ et le $7 \mathrm{o}^{\mathrm{e}}$ jour de la vie foetale. Leur nombre total augmente exponentiellement pendant cette période comme cela est propre à toute multiplication cellulaire. Cette augmentation du nombre de mitoses goniales se produit même après l'apparition des premières figures leptotènes de la prophase méïotique.

Ce fait implique qu'à partir dit $50^{\mathrm{e}}$ jour les mitoses goniales bloquées proviennent à la fois de la poussée de division des gonies-souches qui se poursuit et de la poussée de division des gonies de renouvellement qui commence.

On sait que 1'ovogénèse débute pendant la vie embryonnaire chez les Mammifères ; des divisions goniales puis des prophases méiotiques sont visibles dans l'ovaire de très jeunes embryons. Cette ovogénèse est bientôt suivie de la dégénérescence des ovocytes en prophase méiotique.

La signification et 1'importance de ces deux phénomènes, en eux-mêmes, et par rapport à la structure et au fonctionnement de l'ovaire adulte nous échappent à peut près complètement.

Telle est la raison des recherches que nous poursuivons sur l'ovogénèse chez les Mammifères domestiques.

La colchicine, en bloquant les mitoses en métaphase, permet de mieux analyser les multiplications cellulaires (EIGSTr et DUSTIN, I955) et d'amplifier des variations d'activité dans des ovaires d'âges différents (technique utilisée par CrERMonT et LEBLOND (I953) pour l'étude de la spermatogénèse chez le Rat).

\section{I. - MATÉRIEI, ET MÉTHODES}

Nous avons injecté de 0,175 à $0,225 \mathrm{mg}$ de colchicine par $100 \mathrm{~g}$ de poids vif à 50 embryons de brebis de race Ile-de-France âgées de 35 à 70 jours.

L'injection est faite dans les masses inusculaires postérieures de l'embryon. La grande place tenue par le foie fœtal dans la cavité générale rend impossible une injection intrapéritonéale. 
L'ouverture de l'utérus maternel, aussi près que possible de la poche amniotique répérée au toucher ou par transparence, permet une injection précise ; tout traumatisme et perte amniotique est évitée au maximum par l'utilisation d'aiguilles très fines (40-4/I0). La colchicine pure (MERCK, Darmstadt) est injectée en solution dans le sérum physiologique et sous faible volume: de $0,05 \mathrm{~cm}^{3}$ à $0,2 \mathrm{~cm}^{3}$ suivant la taille du fotus. Ces solutions qui s'oxydent facilement sont préparées extemporanément.

Nous avons toujours opéré au même moment de la journée, Io $h$ à I $2 h$, pour éviter que le rythme journalier des mitoses (BulLough, I948), ne soit un facteur supplémentaire de variation.

Les ovaires ont été fixés au Bouin-Hollande, $4 \mathrm{~h}$ après l'injection. Les coupes sériées de $7,5 \mu$ d'épaisseur sont colorées par la réaction de Feulgen, le conjonctif étant mis en évidence par l'Alcian blue.

\section{II. - RÉSULTATS}

Les figures I et 2 sont les diagrammes de distribution du nombre total de métaphases goniales bloquées dans les ovaires des embryons traités, diagrammes établis en fonction de l'âge (I) et dù poids des embryons (2).

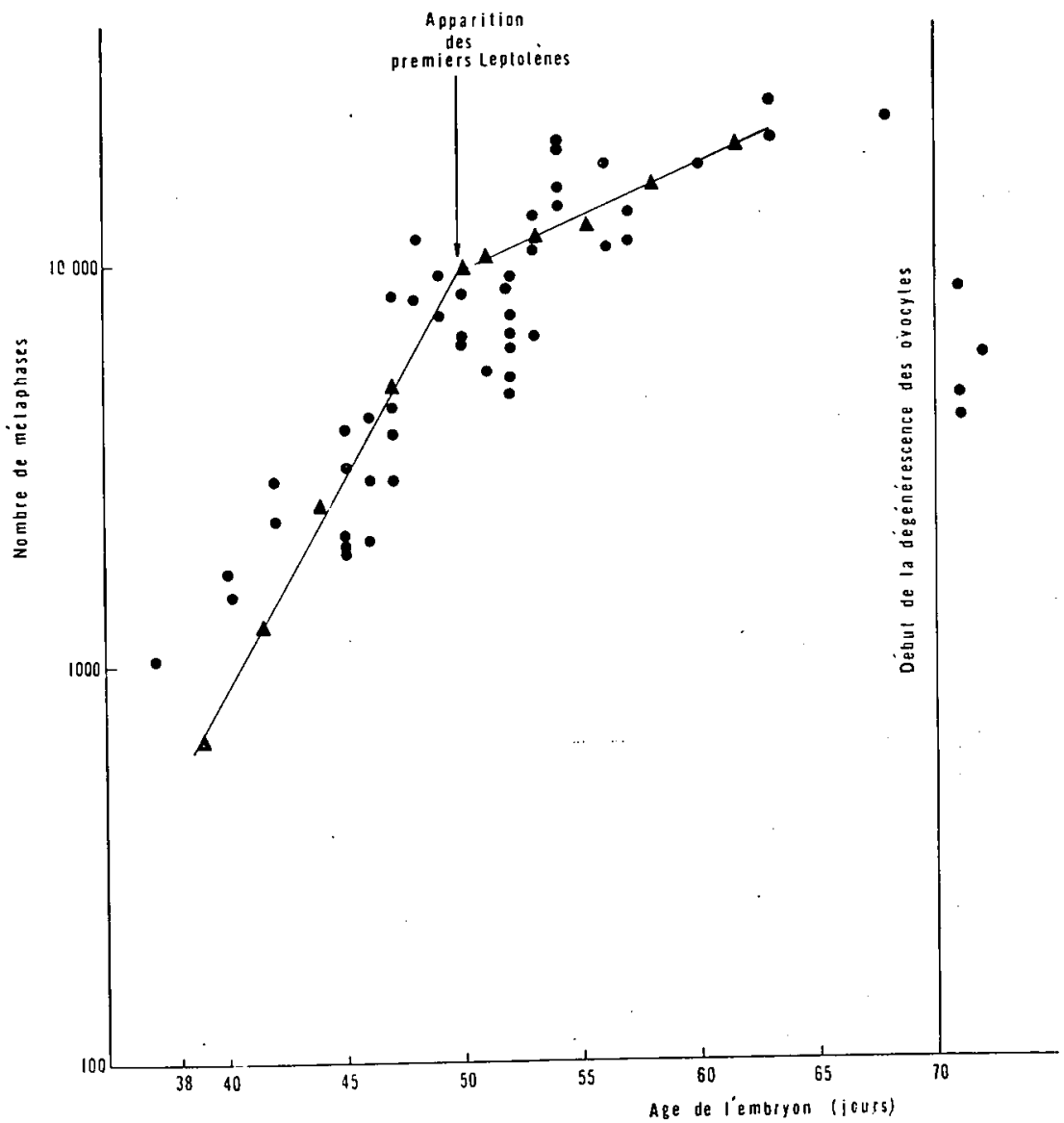

FIG. I. - Diagranme de distribution du nombre de métaphases goniales bloquées par la colchicine établi en fonction de l'âge des embryons dè Brebis (en coordonnées semi-logarithmiques).

(Graphique théorique des variations du nombre de mitoses goniales en fonction de l'âge, tracé en supposant que la " première " poussée goniale n'est pas terminée quand la "deuxième "commence. 
Ils montrent l'existence d'une relation linéaire entre le logarithme du nombre de métaphases goniales et l'âge et, surtout, le poids de l'embryon. La droite de distribution présente un brusque changement de pente au $50^{\mathrm{e}}$ jour de la gestation (pour un poids de $I 8 \mathrm{~g}$ de l'embryon).

Histologiquement, on constate l'apparition pour la première fois dans des ovaires de cet âge dés stades leptotènes de la prophase méiotique.

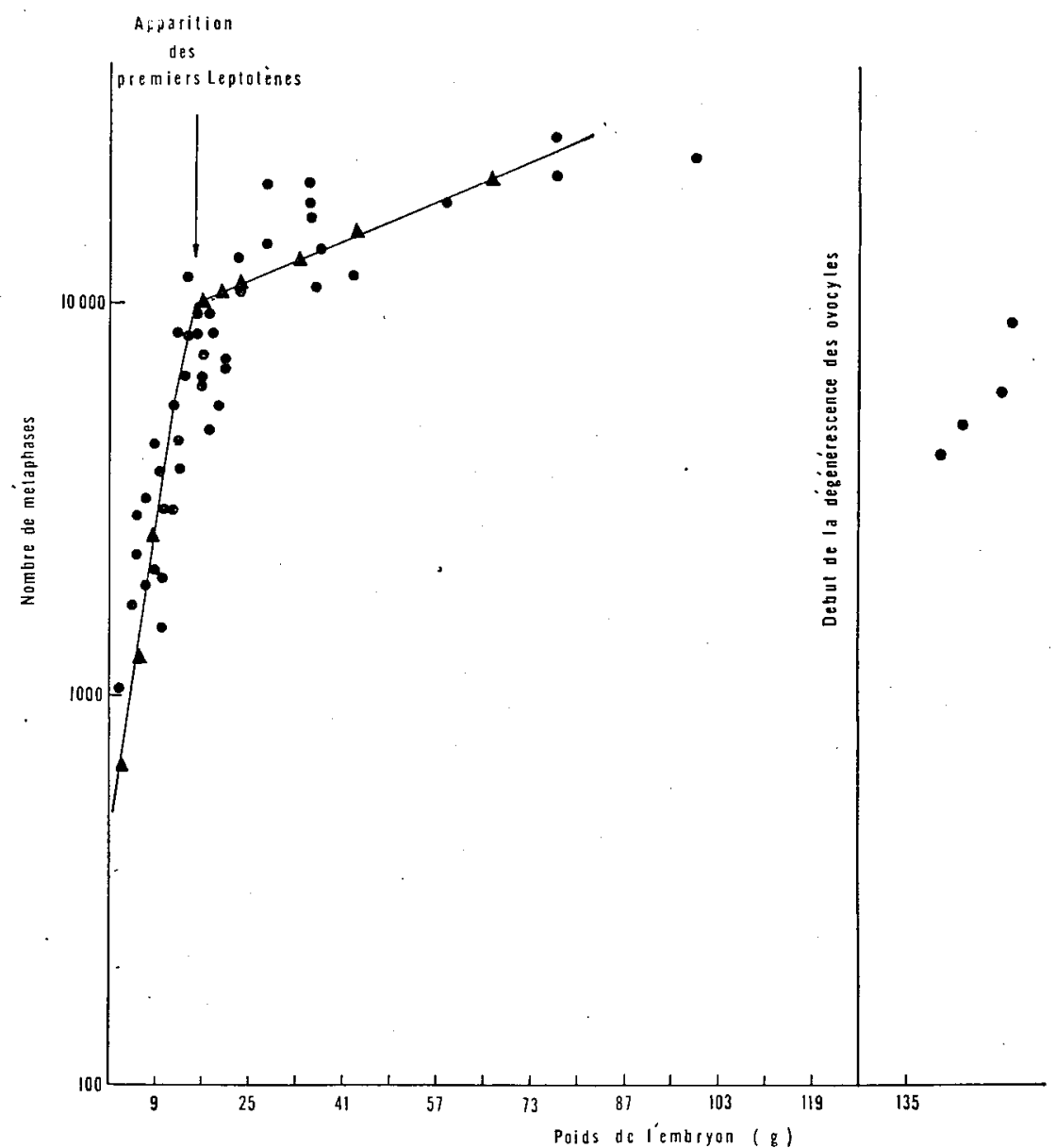

FIG. 2. - Diagramme de distribution du nombre de melaphases goniales bloquées par la colchicine, établi en fonction du poids des embryons de Brebis (en coordonnées semi-logarithmiques).

(Graphique théorique des variations du nombre de mitoses goniales en fonction du poids, tracé en supposant que la " première " poussée goniale n'est pas terminée quand la "deuxième " commence).

L'interprétation de ces résultats est délicate. Plusieurs hypothèses peuvent être envisagées; nous les développerons dans un mémoire ultérieur.

I'une seule d'entre elles permet d'obtenir une bonne concordance entre courbe théorique et le diagramme de distribution des mitoses goniales (fig. I et 2). E1le consiste à admettre l'existence de deux poussées de divisions goniales entre $1 \mathrm{e} 35^{\mathrm{e}}$ et le $70^{\mathrm{e}}$ jour de la gestation. Ces deux poussées se superposent dans le temps. En effet lorsqu'apparaissent les premiers stades leptotènes dans les ovocytes issus de la pre- 
mière poussée, toutes les gonies-souches ne sont pas encore divisées, alors que des gonies de renouvellement mises en réserve à partir des premières gonies-souches entrées en activité d'une manière identique à ce qui se passe au cours de la spermatogénèse (OR'TAVANT, I959) commencent, elles, à se multiplier.

Reçu en mars 1960.

\section{SUMMARY}

COLCHICINE TREATMENT FOR THE STUDY OF GONIAL MITOSES IN EMBRYONIC OVARY OF THE EWE. ANALYSIS OF SOME RESULTAT

Colchicine was used to atrest gonial division in the ovary of the ewe between the $35^{\text {th }}$ and the 7 oth day of fotal life. Their total number increases exponentially during this period as is normal of all cell-multiplication. This increase in the number of gonial mitosis occurs even after the appearance of the first leptotene figures of the meiotic prophase.

This implies that from the 5oth day the blocked gonial mitosis arise both from the cycle of division of the stem cells which follows and from the cycle of division of the renewal cells which is beginning.

\section{RÉFÉRENCES BIBLIOGRAPHIQUES}

BuLLough W.S., 1948. Mitotic activity in adult male mouse, Mus Musculus L. : the diurnal cycles and their relation to waking and sleeping. Proc. Roy. soc. London B, 135, $212-233$.

ClERMONT X. LEBLond C. P., I953. Renewal of spermatogonia in the rat. Amer. J. Anat., 93, 475-50r. EIGSTI O. J., DusTIN Jr P., I955. Colchicine in agriculture, medicine, biology and chemistry. The Iown State College Press, Ames, Iowa, U.S. A.

ORTavant R., 1959. Le cycle spermatogénétique chez le Jélier. Ann. Zoolech., 8, i 85-244. 